\title{
Best Fit Wavelet Function for Path Loss Prediction in Wireless Communication System
}

\author{
Kishor K. Pandyaji \\ PVPIT, Budhgaon \\ Shivaji University, Kolhapur
}

\author{
S.K. Bodhe, PhD \\ Department of Electronics, \\ Pune University
}

\begin{abstract}
The site-specific or statistical channel models can be precisely characterize the propagation channel, their parameters depend on channel statistics and accurate database, making them difficult for implementation. In mobile communication system, a simple model with few parameters to estimate or transmit is of great interest. A new model is proposed using both Fourier and Wavelet transform as a decomposition basis for outdoor propagation. The path loss is decomposed on wavelet packet basis function resulting in scaling and wavelet coefficients. Hard thresholding is used to compress these coefficients as much as possible. Different threshold levels have been tested to find the mean square error (MSE) due to reconstructed path loss after compression. Propagation models for mobile communication system have been evaluated and compared based on this modeling scheme.
\end{abstract}

\section{General Terms}

Propagation model, Path loss, Wavelet Transform, Decomposition and Reconstruction algorithms

\section{Keywords}

Propagation model, Path loss, Best fit Wavelet function, Hard and soft thresholding

\section{INTRODUCTION}

In real world, signals are non-periodic, noisy, intermittent, transient, time variant in nature. The properties of such signal cannot be easily detected. It is most natural and effective to represent these signals by localized finite energy basis. This leads to transformation of signal into another domain. Nonperiodic features can be resolved into much more complex spectrum of frequencies, called Fourier transform [FT]. FT is the transformation of time domain to frequency domain. Fourier coefficients that computed in transformation process are used to analyze the frequencies. But, signal cannot be simultaneously localized in time and frequency. There are two methods that attempt to provide information in both time and frequency: Windowed Fourier Transform [WFT] and the Continuous Wavelet transform. This transformation maps a time function into function of time and frequency.

An attempt has made to analyze and evaluate the performance of outdoor propagation models in transformed domain for wireless communication systems.

\subsection{Fourier Transforms}

Fourier analysis has been the major mathematical tool for signal representation and a processing. The discrete version this approach is called Fourier series, breaks down a given signal into sinusoidal function with different harmonics of the fundamental frequency. Since sinusoidal signal are periodic signal, Fourier analysis is an excellent tool for analyzing this class of signals. However it is inefficient for transient signals. Fourier transform of an analog function $f(t)$ is defined as $f(w)=\int_{-\infty}^{+\infty} f(t) \cdot e^{-j w t} d t$

If $f(t)$ is replaced by delta function $\partial\left(t-t_{0}\right)$ at $t=t_{0}$, then FT becomes a sinusoidal function, hence it takes an infinite number of frequencies to represent the signal that exit only at one point in the time domain.

On the other hand, if the function to be analyzed is a sinusoidal function of a single frequency, a spectrum is delta function. This leads to discrete Fourier transform which is very efficient for studying global periodic function.

\section{WAVELET TRANSFORM}

Wavelets are finite-energy function with localization properties that can be very efficiently to represent transient signals. Efficiency means only finite numbers of coefficients are needed to represent a complicated signal. In contrast with the sinusoidal function of infinite extent, 'wavelet' implies a small wave. Mathematically, the area under the graph of the Wavelet $\psi(t)$ is zero, i.e. $\int_{-\infty}^{+\infty} \psi(t) d t=0$.

In spectral domain, this property is equivalent to $\hat{\psi}(t)=0$, it means the spectrum of the wavelet has a value of zero at $w=0$. In other word; the wavelet has no dc offset. The spectral domain behavior makes the wavelet a band pass filter. It is from any wavelet and its spectrum that energy of wavelet is concentrated in a certain region of both $t$ and axles. This localization property is an important feature of wavelets. If the wavelet is more localized it produces better (higher resolution) representation of signal in the time-frequency plane and require fewer coefficients in the representation.

\subsection{Wavelet at Different Resolution (Scales)}

For a given wavelet $\psi(t)$, a scaled and translated version is designated by

$\psi_{b a}(t)=\frac{1}{\sqrt{a}} \psi\left(\frac{t-b}{a}\right)$

The parameter ' $a$ ' corresponds to the scale while $\mathrm{b}$ is the translation parameter. The wavelet $\psi_{01}(t)=\psi(t)$ is called the basic wavelet mother wavelet.

It is important to note that the shape of the wavelet remains the same under translation and scaling. The wavelet signal processing is not much different from that of Fourier processing, at least when 'Orthogonal Wavelet' are considered. Instead of decomposing a signal into sinusoidal functions of different frequencies, wavelet signal processing 
seeks to decompose a transient signal into a linear combination of the scaled and translated version of the basic wavelet.

\subsection{Continuous Wavelet Transform}

The continuous (integral) wavelet transform (CWT/IWT) of a signal $x(t)$ is a linear transform defined by the integral,

$$
\begin{aligned}
W_{\psi} x(b, a) & =\int_{-\infty}^{+\infty} x(t) \overline{\psi_{b a}(t)} d t \\
W_{\psi} x(b, a) & =\frac{1}{\sqrt{a}} \int_{-\infty}^{+\infty} x(t) \overline{\left(\frac{t-a}{a}\right)} d t \\
& =\left\langle x(t), \psi_{b a}(t)\right\rangle
\end{aligned}
$$

The last expression in above equation represents the inner product of two functions, defined as,

$$
\langle f, g\rangle=\int_{-\infty}^{+\infty} f(t) \overline{g(t)} d t \text {. }
$$

The CWT computes the wavelet coefficient of $x(t)$ associated with the wavelet $\psi_{b a}(t)$.This coefficient gives correlation between these two functions. Higher correlation produces a larger coefficient.

The original signal is uniquely recovered by double integral is given by

$$
x(t)=\frac{1}{C_{\psi}} \int_{-\infty}^{+\infty} \int_{-\infty}^{+\infty} W_{\psi}(x)(b, a) \psi_{b a}(t) \frac{d b d a}{a^{2}}
$$

Where, $C_{\psi}$ is a finite constant given by the integral

$$
C_{\psi}=\int_{-\infty}^{+\infty} \frac{|\widehat{\psi}(w)|^{2}}{|w|} d w<\infty
$$

\section{DECOMPOSITION AND RECONSTRUCTION ALGORITHMS}

The decomposition (analysis) and reconstruction (synthesis) algorithms are the most often used algorithm in wavelet signal processing. It consists of simply dividing the signal into components so that each component may be proceeding with a different algorithm. The important issue of these algorithms is to be able to reconstruct the signal perfectly on application all-pass filter for all signal components. These algorithms are based on two scale relation.

\subsection{Decomposition of a 1-D Signal}

The Decomposition algorithm for scaling function coefficients is described by

$$
C_{j k}=\sum_{\ell} a_{2 k}-\ell C_{j}+1, \ell
$$

By rewriting same equation as,

$$
C_{j k}=\sum_{\ell}\left(a_{m-\ell}\right) \mid m=2 k C_{j}+1, \ell
$$

This is interpreted as convolution before down sampling by 2 . The computation of the wavelet coefficients at one level of resolution is carried out in similar manner, namely

$$
D_{j, k}=\sum_{\ell}\left(b_{m-\ell}\right) \mid m=2 k C_{j}+1, \ell
$$

These two steps are combined to form decomposition block. This decomposition block can be repeated and sequentially applied to the scaling function coefficients to yield the wavelet coefficient sequences. Implementation of $C_{j k}$ is simple. Taking a scaling coefficient set to convolve with coefficient set $\{a k\}$ and down sampling yields the scaling function coefficients at one lower resolution level. Repeating same procedure with coefficient set $\{b k\}$ yields the wavelet coefficients. These procedures are repeated to yield the coefficient oat lower resolution levels.

\subsection{Reconstruction of 1-D Signals}

The two scale relation for the approximation space and the wavelet space constitute te reconstruction algorithm. The scaling function coefficients at a higher resolution level are computed by using the formula

$$
C_{j+1, \ell}=\sum_{k}\left(p_{\ell-2 k} C_{j, k}+q_{\ell-2 k} d_{j, k}\right) \text {. }
$$

Each summation can be interpreted as a convolution process after up-sampling. It can be repeated for coefficient sequences $\left\{C_{j-p, k}\right\}$ and $\left\{d_{j-p, k}\right\}, p=M, M-1, \ldots 0$.

\subsection{Thresholding}

Thresholding is one of the most often used processing tools in wavelet signal processing. It is used in nose reduction, in signal and image compression, and sometimes in signal reorganization. There are different types of thresholding in use. The choice of thresholding methods depend on the application are briefly discussed below,

\section{Hard Thresholding}

Hard thresholding is sometimes referred as gating. If a signal (or a coefficient) value is below a preset value, it is set to zero.

$y= \begin{cases}x & \text { for all }|x| \geq \kappa \\ 0 & \text { otherwise }\end{cases}$

Where, $\kappa$ is the threshold value or gate value.

\section{Soft Thresholding}

It is defined as

$y=\left\{\begin{array}{l}f(x-\kappa), \quad \text { for all }|x| \geq \kappa \\ 0 \quad \text { otherwise }\end{array}\right.$

The function $f(x)$ generally is a linear function. However, spline curves of third and fourth orders may be used to effectively weight the value greater than $\kappa$. 


\section{MODELING SCHEME}

A new model is proposed using both Fourier and Wavelet transform as a decomposition basis outdoor propagation models like Hata, COST, Lee, Egli, Xia, SUI, Ray models[18]. The path loss is decomposed on wavelet packet basis function resulting in scaling and wavelet coefficients. Hard and soft thresholding is used to compress these coefficients as much as possible. Different threshold levels have been tested to determine the mean square error (MSE) due to reconstructed path loss after compression [13].

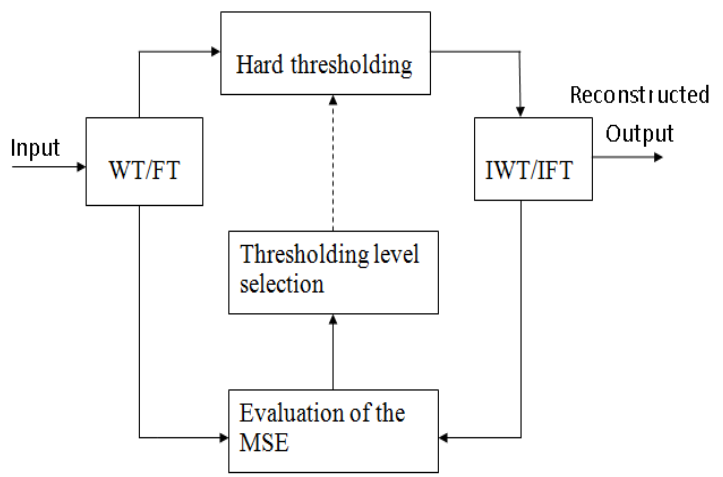

Figure 1: Transformed Domain Modeling Scheme

The performance parameter, Root Mean Squared Error,

$$
\begin{aligned}
\text { RMSE }= & \sqrt{M S E}, \\
M S E= & \frac{1}{N} \sum_{n=1}^{N}\left|L_{\text {comp }}-L_{\text {pred }}\right| \\
& N \text { - Length of } L_{p} \text { vector }
\end{aligned}
$$

\section{SIMULATION RESULTS}

The Path loss prediction model is proposed using Wavelet transform. The different wavelets namely Symlets, Daubechies, Coiflets and Biorthogonal, are used in design of proposed path loss model for wireless communication system. The path loss computed is decomposed on wavelet packet basis function. Hard and soft thresholding is used to compress these coefficients as much as possible. Different threshold levels have been used to test the predicted path loss on the basis of RMSE (Root Mean Squared error).

\section{CASE I}

To find the appropriate mother wavelet, the experiments are carried out extensively with all wavelets family. For this purpose 2-ray model is considered as case[10,12]. The 2-ray model is embedded by 'synN', 'dbN', 'coifN', and 'biorN' wavelets with thresholding. The model is analyzed on both hard thresholding and soft thresholding scheme.

In analysis, it is found that 'sym8' is the appropriate from Symlet family. The wavelets 'db4' and 'db8' are equally well from Daubechies family and ciof4' is most appropriate wavelets from Coiflets wavelet family. The wavelet 'bior4.4' is the best choice from Biorthogonal wavelets For 2-ray model:

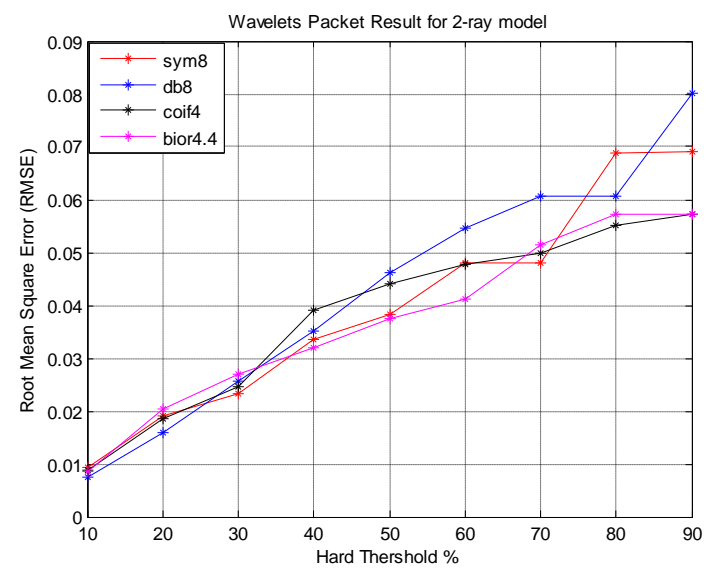

Figure 2: RMSE vs. Soft Threshold Using sym8, db8, coif4 and bior4.4

Table 1: RMSE values for 2-Ray Model

\begin{tabular}{|l|l|l|l|}
\hline Hard threshold & $10 \%$ & $50 \%$ & $90 \%$ \\
\hline Smy8 & 0.0092 & 0.0384 & 0.0691 \\
\hline Db8 & 0.0076 & 0.0461 & 0.0802 \\
\hline Coif4 & $\mathbf{0 . 0 0 8 8}$ & $\mathbf{0 . 0 4 4 1}$ & $\mathbf{0 . 0 5 7 2}$ \\
\hline Bior4.4 & 0.0086 & 0.0375 & 0.0573 \\
\hline
\end{tabular}

Table 1 shows RMSE values at minimum (10\%) to maximum $(90 \%)$ using both hard and soft threshold the wavelet. In all 'coif4' is best fit on hard threshold for the 2-ray model.

\section{CASE II}

In second phase, best possible wavelets are applied for different outdoor propagation models [1-11] by varying threshold value from $10 \%$ to $90 \%$ with increment of $10 \%$. Simulation is carried out for well known propagation models which include empirical, deterministic or site specific and theoretical models. Results have been observed for these models and represented in terms of the evaluation parameter RMSE (Root mean square error). The performances of models are highlighted in tabular form and represented in tgraphically in figures $(3,4,5,6$.and 7$)$.

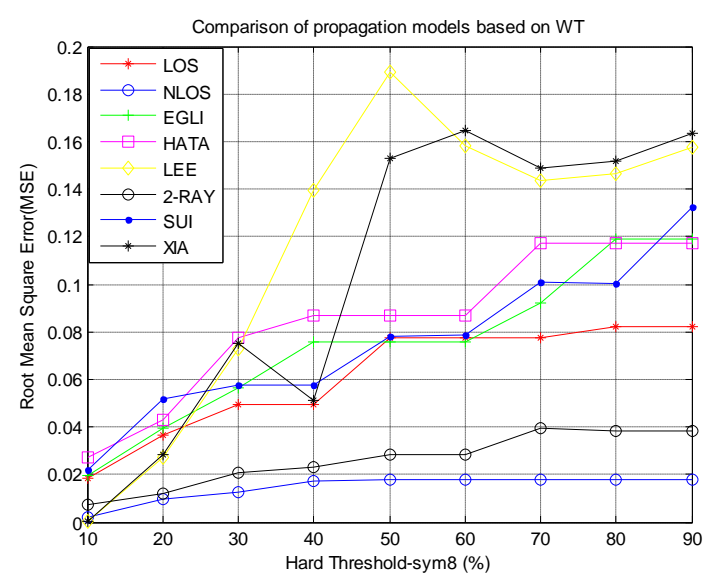

Figure 3: RMSE vs. Hard Threshold using sym8 


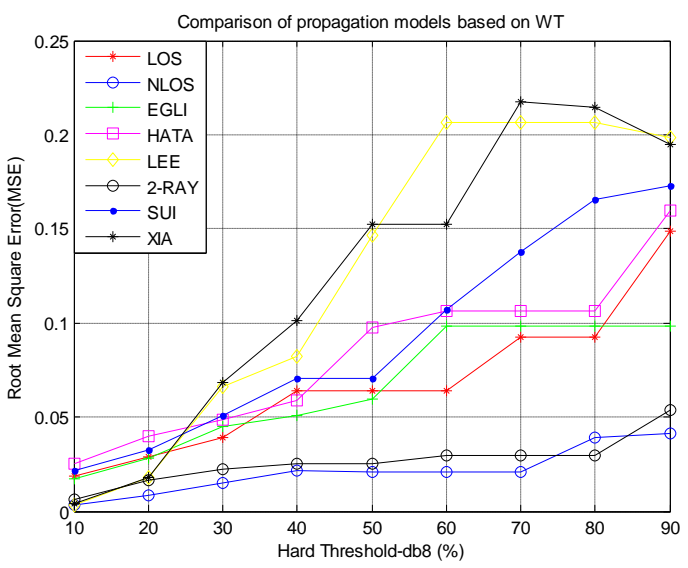

Figure 4: RMSE vs. Hard Threshold using db8

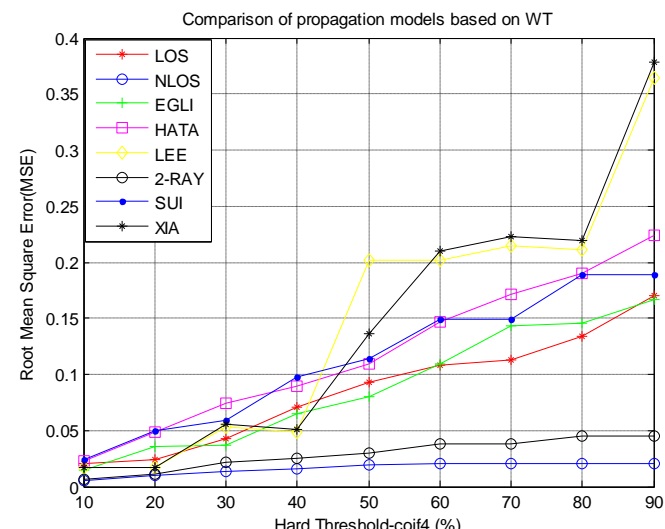

Figure 5: RMSE vs. Hard Threshold using coif4

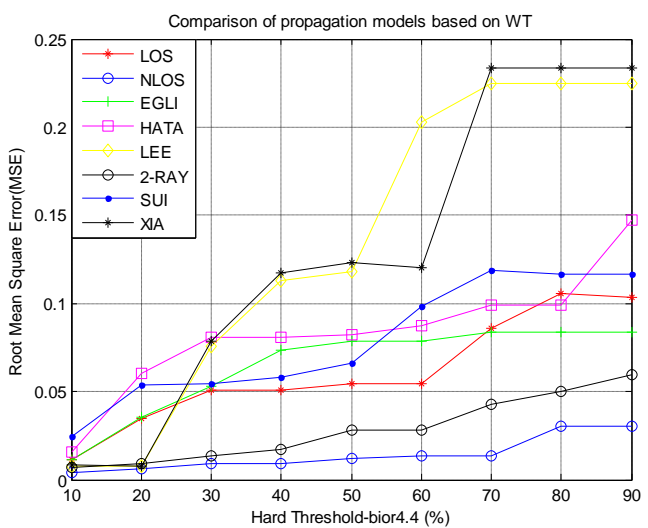

Figure 6: RMSE vs. Hard Threshold using bior4.4

For LOS COSTWI model:

Table 2: RMSE values for LOS COSTWI Model

\begin{tabular}{|c|c|c|c|}
\hline \multirow{2}{*}{ Wavelet } & \multicolumn{3}{|c|}{ Hard threshold } \\
\cline { 2 - 4 } & $10 \%$ & $50 \%$ & $90 \%$ \\
\hline Smy8 & 0.0040 & 0.0121 & 0.0228 \\
\hline Db8 & 0.0044 & 0.0140 & 0.0328 \\
\hline Coif4 & 0.0050 & 0.0137 & 0.0234 \\
\hline Bior4.4 & 0.0042 & 0.0094 & 0.0311 \\
\hline
\end{tabular}

The RMSE obtained from 'sym 8 ' is constant over $30 \%$ to $80 \%$ thresholding. The 'bior 4.4 ' wavelet is applicable up to $50 \%$ threshold value for LOS COSTWI model.
For NLOS COSTWI model:

Table 3: RMSE values for NLOS COSTWI Model

\begin{tabular}{|l|l|l|l|}
\hline \multirow{2}{*}{ Wavelet } & \multicolumn{3}{|l|}{ Hard threshold } \\
\cline { 2 - 4 } & $10 \%$ & $50 \%$ & $90 \%$ \\
\hline Smy8 & 0.0023 & 0.0177 & 0.0177 \\
\hline Db8 & 0.0030 & 0.0206 & 0.0411 \\
\hline Coif4 & 0.0058 & 0.0197 & 0.0200 \\
\hline Bior4.4 & 0.0042 & 0.0118 & 0.0306 \\
\hline
\end{tabular}

The wavelet 'sym8' is the best choice for NLOS COSTWI model.

For Egli model:

Table 4: RMSE values for Egli Model

\begin{tabular}{|l|l|l|l|}
\hline \multirow{2}{*}{ Wavelet } & \multicolumn{3}{l|}{ Hard threshold } \\
\cline { 2 - 4 } & $10 \%$ & $50 \%$ & $90 \%$ \\
\hline Smy8 & & 0.0757 & 0.1188 \\
\hline Db8 & 0.0174 & 0.0597 & 0.0981 \\
\hline Coif4 & 0.0147 & 0.0800 & 0.1665 \\
\hline Bior4.4 & 0.0116 & 0.0787 & 0.0836 \\
\hline
\end{tabular}

The wavelet 'Bior4.4' is the best fit for NLOS COSTWI model.

For Hata model:

Table 5: RMSE values for Hata Model

\begin{tabular}{|l|l|l|l|}
\hline \multirow{2}{*}{ Wavelet } & \multicolumn{3}{|l|}{ Hard threshold } \\
\cline { 2 - 4 } & $10 \%$ & $50 \%$ & $90 \%$ \\
\hline Smy8 & 0.0270 & 0.866 & 0.1173 \\
\hline Db8 & 0.0249 & 0.0977 & 0.1601 \\
\hline Coif4 & 0.0228 & 0.1089 & 0.2241 \\
\hline Bior4.4 & 0.0158 & 0.0821 & 0.1469 \\
\hline
\end{tabular}

The wavelet 'Bior4.4' is the best suit up to $80 \%$ threshold for NLOS COSTWI model

For LEE model:

Table 6: RMSE values for Lee Model

\begin{tabular}{|l|l|l|l|}
\hline \multirow{2}{*}{ Wavelet } & \multicolumn{3}{l|}{ Hard threshold } \\
\cline { 2 - 4 } & $10 \%$ & $50 \%$ & $90 \%$ \\
\hline Smy8 & 0.0002 & 0.1894 & 0.1576 \\
\hline Db8 & 0.0036 & 0.1467 & 0.1984 \\
\hline Coif4 & 0.0165 & 0.2020 & 0.3641 \\
\hline Bior4.4 & 0.0078 & 0.1183 & 0.2247 \\
\hline
\end{tabular}

The RMSE obtained from 'Bior4.4' increases abruptly above $50 \%$ thresholding (hard), it is admissible up to $50 \%$ threshold For SUI model:

Table 7: RMSE values for SUI Model

\begin{tabular}{|l|l|l|l|}
\hline \multirow{2}{*}{ Wavelet } & \multicolumn{3}{|l|}{ Hard threshold } \\
\cline { 2 - 4 } & $10 \%$ & $50 \%$ & $90 \%$ \\
\hline Smy8 & 0.0156 & 0.0995 & 0.1165 \\
\hline Db8 & 0.0248 & 0.1365 & 0.1996 \\
\hline Coif4 & 0.0250 & 0.0951 & 0.1367 \\
\hline Bior4.4 & 0.0220 & 0.0561 & 0.0981 \\
\hline
\end{tabular}


The wavelet 'Bior4.4' is the appropriate choice for SUI model.

For Xia model:

Table 8: RMSE values for Xia Model

\begin{tabular}{|l|l|l|l|}
\hline \multirow{2}{*}{ Wavelet } & \multicolumn{3}{|l|}{ Hard threshold } \\
\cline { 2 - 4 } & $10 \%$ & $50 \%$ & $90 \%$ \\
\hline Smy8 & 0.0002 & 0.1529 & 0.1636 \\
\hline Db8 & 0.0038 & 0.1525 & 0.1948 \\
\hline Coif4 & 0.0172 & 0.1357 & 0.3783 \\
\hline Bior4.4 & 0.0837 & 0.3474 & 0.5725 \\
\hline
\end{tabular}

The performance of 'sym8' is good on hard threshold and 'bior4.4' is better choice on soft threshold.

\section{CASE III:}

The Path loss prediction model is proposed on basis of Fourier transform. The computed path loss is decomposed by FFT (Fast Fourier transformation) and reconstructed by inverse Fourier transformation. Different threshold levels have been applied to find RMSE (Root Mean Squared error) due to the reconstructed path loss. RMSE achieved by simulation indicates that the performance of FT basis modeling is very poor.

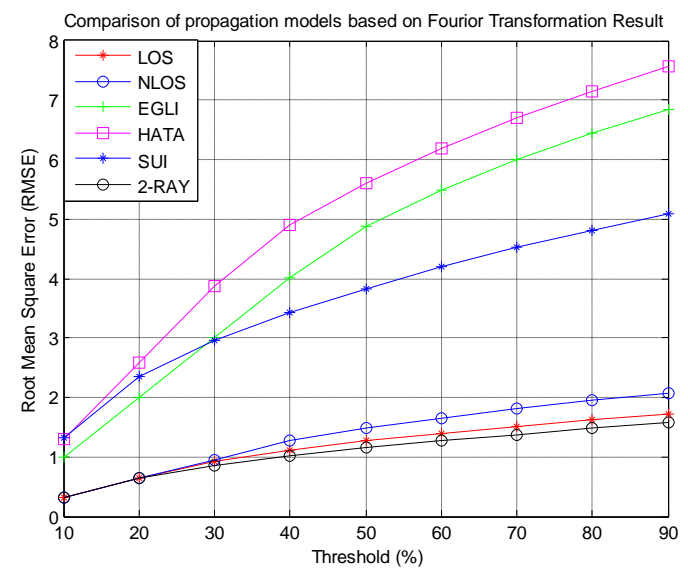

Figure 7: RMSE vs. Threshold using Fourier Transform

\section{CONCLUSION}

In analysis, best fit mother wavelet is determined from wavelet family. The performance of propagation models are evaluated using best fit wavelets. FT is also used to evaluate the performance of propagation model. Best fit wavelets have good accuracy over the $10 \%$ to $50 \%$ threshold and moderate above $50 \%$ to $90 \%$ threshold. Coiflet wavelets performed well in deterministic model like ray model. For empirical model like Hata and Egli, the Biorthogonal wavelet is the excellent choice. COSTWI LOS \& NLOS is the theoretical model, Symlet wavelet is better option. Physical models like Xia model are based on physical optics. The Symlet or Biorthogonal wavelets are reasonably good. In comparison with FT, WT is promising mathematical tool in modeling.

\section{ACKNOWLEDGMENTS}

Our thanks to PVPIT research cell and faculties of department of Electronics

\section{REFERENCES}

[1] H. Mata, "Empirical Formula for Propagation Loss in Land Mobile Radio Services," IEEE Transaction on Vehicular Technology, VT-29, No.3, pp.317-325, Aug.1980.

[2] J. J. Egli, "Radio Propagation above $40 \mathrm{Mc}$ Over Irregular Terrains," Proc. IRE. Vol. 15.No.10, pp.13831391 , Oct.1957.

[3] "COST: Urban Transmission Loss Models for Mobile Radio in the 900 and $1800 \mathrm{MHz}$ Bands," Rep. COST 231 $T D$ (91) 73, the Hague Netherlands, Sept.1991.

[4] Gilles Y. Delisle et al., "Propagation Loss Prediction: A Comparative Study with Application to the Mobile Radio Channel," IEEE Transaction on Vehicular Technology, VT-34 No.2, pp. 86-92, May.1985.

[5] W.C.Y. Lee, "Lee's Model," IEEE Transactions on Vehicular Technology, Vol. 31, No.1, February 1988.

[6] V. Erceg, K. V. S. Hari et al., "Channel Models for Fixed Wireless Applications," technical report, IEEE 802.16 Broadband Wireless Access Working Group, January 2001.

[7] K. Allsebrook and J. D. Parsons, "Mobile Radio Propagation in British Cities at Frequencies in the VHF and UHF Bands," IEEE Transaction on Vehicular Technology, Vol.26, No.4, pp. 313-323, Nov.1977.

[8] H. H. Xia, H. L. Bertoni, L. R. Maciel, A. LindsayStewart, and R. Rowe, "Radio Propagation Characteristics for Line-of Sight Microcellular and Personal Communications," IEEE Transaction on Antennas and Propagation,Vol.41, pp.1439-1447, Oct.1993.

[9] L. R. Maciel, H. L. Bertoni, and H. H. Xia., "Unified Approach to Prediction of Propagation over Buildings for All Ranges of Base Station Antenna Height," IEEE Transactions on Vehicular Technology, Vol. 42, pp.4145, Feb.1993.

[10] N. Papadakis, Athanasios G. Kanatas, Member, IEEE, and Philip Constantinou, Senior Member, IEEE "Microcellular Propagation Measurements and Simulation at $1.8 \mathrm{GHz}$ in Urban Radio Environment," IEEE Transactions on Vehicular Technology, Vol. 47, No. 3, Aug. 1998.

[11] Howard H. Xia, "A Simplified Analytical Model for Predicting Path Loss in Urban and Suburban Environments," IEEE Transactions on Vehicular technology, Vol.46, No.4, November 1997.

[12] N. Papadakis, "Radio Propagation Measurements and Modeling Using Ray Tracing Techniques,” IEEE, Trans. 1994, pp.1767-1770.

[13] Seyed Mohammad, Sajad Sadough, Emmanuel Jaffrot, "A Wavelet Packet Based Model For An Ultra-WideBand Indoor Propagation Channel" ECPS 2005 Conference, 15-18 March 2005, BREST, FRANCE 